\title{
Hardware Design of Low Energy Consumption Wireless Senor Network Node for Monitoring Mechanical Vibration Signals
}

\author{
Ma Naji1, ${ }^{1,}$, Diao Ming ${ }^{1}, \mathrm{Hu}_{\mathrm{Xiao}}{ }^{3}, \mathrm{Sun} \mathrm{Jian}^{2}$ and Ma Anchang ${ }^{2}$ \\ ${ }^{1}$ Information \& Communication College Harbin Engineering University \\ ${ }^{2}$ Information \&Network Center Heilongjiang University of Science \&Technology \\ ${ }^{3}$ Institude Of Electronics Chinese Academy Sciences \\ 5203datashare@163.Com
}

\begin{abstract}
Aiming at the increasing demand of the monitoring of mechanical vibration signals, by improving the existing Sensor Monitoring Network, a low-power wireless sensor network is designed. The data transmission efficiency is increased by using the compressed sensing (CS) technology to compress the mechanical vibration signals of the devices monitored by the network. To realize real-time processing of monitoring signals, the $F P G A+D S P$ architecture is treated as the core of the equipment processing the mechanical vibration signals of wireless sensor network. The modified designs of the hardware circuit and logic circuit with regard to the data collection, sampling, transmission, processing and the store section are introduced initially. Further, the effects of the improved Sensor Monitoring Network are tested by the experimental study on the rotating equipment. The results show that the real-time processing can be well implemented and the power can be cut down to a certain degree.
\end{abstract}

Keywords: WSN, Monitor, Low Energy Consumption, DSP

\section{Introduction}

Monitoring the mechanical vibration signal of the equipment can effectively forecast its running status, and reduce its hazard rate. When the equipment breaks down, rapid failure diagnosis can be implemented with the help of the monitoring data. Hence monitoring of mechanical vibration of the equipment displays its remarkable significance ${ }^{[1]}$. Traditionally, the cable network is adopted in the existing mechanical vibration monitoring systems, which may lead to the drawbacks of the wiring complexity, lack of flexibility, high costs. Meanwhile, quite a lot of equipments belong to the rotating machineries, so it is difficult to set sensors on the equipments if the wired monitoring network is used. Additionally, the current sensor monitoring network is inferior for its low sampling resolution. Due to the characteristics of the mechanical vibration signals, exceedingly high sampling frequency is required for high-precision signal sampling followed by massive sampling data and direct transmission without signal processing is doomed to dampen down the network transmission efficiency. Furthermore, the monitoring network signal processing presently applied operates generally at a low speed and real-time signal processing seems unrealizable.

In view of the problems from the site survey, the wireless sensor monitoring network (WSMN) for monitored the mechanical vibration of the equipment was designed. It primarily makes improvement of the vibration signal acquisition and transmission modes and lessens the size of sampling vibration signals, which efficaciously heightens the signals transmission efficiency. In the same course, the WSMN can make real-time vibration signal processing come true. As the energy consumption is reduced in the design, batteries can be used as the power supply 
device for the monitoring terminal, which can boost improve the flexibility of the monitoring network.

\section{Overall Hardware Design of Wireless Sensor Monitoring Network (WSMN)}

The WSMN mainly consists of three parts: 1.Monitoring terminal (MT): The MT is responsible for collecting the mechanical vibration signals of the equipment, the signals of A / D conversion, and signals transmission. 2. Mechanical vibration signal processing device (MVSPD): The MVSPD is responsible for reconstructing the compressed vibration signals, signal filtering and noise reduction, signal preprocessing, signal processing, extraction of the information on mechanical vibration of the monitored equipment and storage of the information. 3. Principal computer (PC): The PC is responsible for storage of the mechanical vibration data, sampling control, drawing the parameter curves and data analysis. The structure chart of the WSMN is shown in Figure 1.

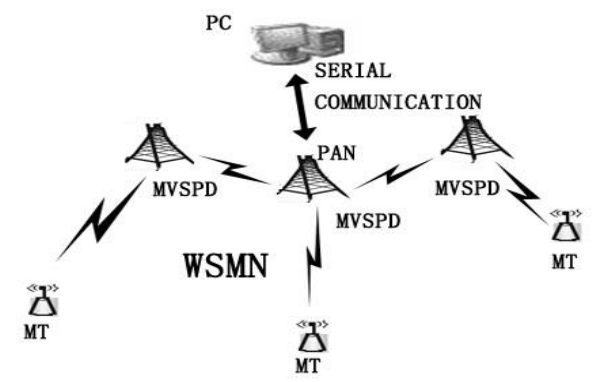

Figure 1. Structure Chart of The Wireless Sensor Monitoring Network

\section{Hardware Design of a Monitoring Terminal}

The MT of the WSMN is tasked with the acquisition, A/D conversion, compression and transmission of the mechanical vibration signals of the monitored equipment. The design of the MT should take into account the its of lower power dissipation and higher energy efficiency and simultaneously ensure the high precision sampling of the vibration signals. Given the above-mentioned characteristics, the schematic diagram of the hardware structure of the MT we design is indicated in Figure 2.

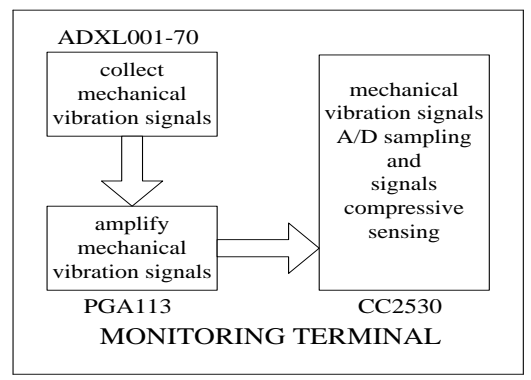

Figure 2. Block Diagram of the Monitoring Terminal's Hardware

The MT is designed by applying the high performance sensor (ADXL01-70 of ADI) to collect the mechanical vibration signals. Such type of sensor boasts the advantages of no requirement for complicated signal conditioning circuit which can cut down the volume and energy consumption of the MT with effects [2]. The mechanical vibration signals outgoing from the sensor are amplified by the programmable amplifier before A/D conversion. The PGA113 is used as the programmable gain amplifier in the design. 
The design of the MT adopts the low energy consumption wireless RFID chip (CC2530 with a frequency band of $2.4 \mathrm{GHz}$ ) as the core to complete the tasks of sampling, compression and transmission. The high-resolution timer controls sampling and compressing in the chip. The high-resolution timer's clock comes from clock division of the CC2530 CPU. The $32 \mathrm{MHz}$ XOSC is used as the clock source in the CPU kernel. When the timer overflows, an interrupt request can be generated. However, the timer will not stop working, but count once again. In this manner, the handling of the interrupt program will not affect the accuracy of the timer, which furnishes the conditions for the rigorous control of the sampled sequence [3]. The MVSPD has greater memory space at the other side of the WSMN, also including the DSP chip and the FPGA chip that can work out the reconstruction algorithm [4]. Additionally, the CC2530 chips back up the IEEE802.15.4 protocol. The integrated microprocessor on the chips can operate the wireless network communication protocol which can flexibly and automatically form the wireless network [5]. The wireless communication protocol is put into utilization on the physical layer to develop such functions as signal acquisition, sampling, transmission and control and materialize the formation of the wireless monitoring network between the MTs and the MVSPDs [6]. When there is no task for vibration signal acquisition, the power supply of the sensor and the A/D conversion circuit can be turned off, only with the wireless network linked. If there is no task for vibration signal acquisition for a long time, CC2530 can also be suspended into hibernation to lower energy consumption [7].

\section{Algorithm of Vibration Signals Compressive Sensing}

Based on the characteristics of mechanical vibration signals, the A/D conversion chip is required to have extremely high sampling frequency to ensure no frequency aliasing in the sampling signals. Meanwhile high sampling frequency consequently generates plenty of sampling data, which will lower the transmission efficiency of the network and the memory spaces. For this reason, the available signal (Data) sampling and transmission approaches have been ameliorated to wipe out this bottleneck. In the circumstances of no influence upon the signal (data) sampling resolution and the information, the sampling signals should be handled through compressive sensing (CS) before the compressed signals transmission so as to effectually reduce the size of data transmission. The MVSPD will reconstruct the original mechanical vibration signals (data) in line with the compressed signals (data) it receives.

Compressive sensing(CS) technology is also named as compressive sampling or sparse sampling, which is a method to seek the sparse solution of the underdetermined linear system brought forward by David Donoho, Emmanuel Candes, Terence Tao ,J. Romberg. in 2004 ( Donoho, 2006 ; Candes,2006a, c, d).

The CS predominantly includes three steps: 1 . signal sparse decomposition; 2. linear measurement of sparse signal; 3. sparse signal reconstruction from a small number of measurements [8]. Through processing the size of transmitted data can be efficaciously brought down.

\subsection{Sparse Decomposition of Mechanical Vibration Signals}

As in this heading, they should be Times New Roman 11-point boldface, initially capitalized, flush left, with one blank line before, and one after.

Suppose the discrete signal of the actual value of the MT (i) is $x$, which can be regarded as a dimensional vector quantity and suppose there is a basis in $n$ dimension linear space, $W=\left[w_{1}, w_{2}, w_{3}, \cdots, w_{N}\right]$ and it is satisfied that $W \cdot W^{T}=W^{T} \cdot W=I$ ( $I$ represent unitary matrix). The basis of the vibration signals can be expressed as follows: 


$$
x=\sum_{i} S_{i} \cdot w_{i}
$$

In the formula, $s_{i}$ is the inner product of vector $x$ and vector ${ }_{w_{i}}$, namely:

$$
S_{i}=\left\langle w_{i} \cdot x\right\rangle
$$

From Formula (1), it can be obtained that:

$$
S=W^{T} \cdot x
$$

Just because it is the orthogonal matrix whose transposed matrix is identical with its inverse matrix, the vibration signal $x$ can be expressed as follows:

$$
x=W \cdot S
$$

There into, $S=\left[s_{1}, s_{2}, s_{3}, \cdots, s_{N}\right]$, it is called the sparse coefficient. The sparse feature shows that most elements in $S$ are zero or similar to zero (this is because the engineering design also needs to go through zero processing). Suppose there are $k$ non-zero elements in $S(k \square N)$, we name it the sparseness of $S$.

\subsection{Linear Measurement of Sparse Signals}

As in this heading, they should be Times New Roman 11-point boldface, initially capitalized, flush left, with one blank line before, and one after.

The perception matrix is the subject of CS as a method to acquire the compressed signal. Suppose there is a matrix $A, A \in R^{M \times N}$ 。 Linear compressed signals $y\left(y \in R^{M}\right)$ can be obtained by means of linear projection of the vibration signal $x$ from $\mathrm{N}$-dimensional linear space to the $\mathrm{M}$-dimensional space $\left.{ }_{(N} \succ_{M}\right)$ with the help of matrix $A$ :

$$
y=A \cdot x
$$

Now the $y$ includes all the information about the original the signal $x$. In the formula the $A$ is named as sensing matrix. In the theory of CS, the measurement is a non-adaptive process. That is to say, the matrix $A$ does not need to change with the change of the signal $x$ but requires that the sensing matrix must comply with the Restricted Isometric Property (RIP) (Candes 2008). RIP is applied to depict the degree of non-orthogonal that approximates the orthogonal matrix with its definition listed below [9]:

Suppose, The $U$ is an integer in which $1 \square U \square N$. If there is an integer $\delta$ that satisfies any sub matrix of $M \times U$ and each vector of the matrix, then:

$$
(1-\delta) \cdot \square y \square_{2}^{2} \leq \square A \cdot y \square_{2}^{2} \leq(1+\delta) \cdot \square y \square_{2}^{2}
$$

What's more, apart from the restriction of the sensing matrix over the degree of non-orthogonal, it must be ensured that there is no correlation between the sensing matrix $A$ and the sparse matrix $W$. Nonetheless, it is fairly difficult to find two completely uncorrelated matrixes in practical design. In this case, we need to select the appropriate the sparse matrix and the sensing matrix by means of calculating the relevance of the two matrixes.

Select a column vector $w_{j}\left(w_{j} \in R^{N}\right)$ and $a_{K}\left(a_{k} \in R^{N}\right)$ respectively from the sparse matrix $W$ and the sensing matrix $A$, then: 


$$
\mu(A, W)=\sqrt{N} \cdot \max \left|\left\langle w_{j}, a_{k}\right\rangle\right|
$$

The $\mu$ is referred to as the relevancy of the two matrixes, $\mu \in(1, \sqrt{N})$. For instance, if Haar wavelet basis is chosen as the sparse base and the noiselet matrix as the sensing matrix, then the relevancy $\sqrt{2}$ can be calculated through the large scale sample value [10]. Since Gaussian random matrix is uncorrelated with most matrixes composed of orthogonal bases to meet RIP with a high probability, the design selects Gaussian random matrix $(M \times N)$ as the sensing matrix.

\subsection{Reconstruction of Sparse Signals}

As in this heading, they should be Times New Roman 11-point boldface, initially capitalized, flush left, with one blank line before, and one after.

The signal $y$ is reconstructed at the receiving terminal, that is to say, to thoroughly renew the vibration signal $x$ (N-dimensional) from the received signal $y$ (M-dimensional). However, the formula (5) tells that the reconstruction is a process to find the solution of equations comprising $N$ variables and $M$ equations. Since ${ }_{N \succ M}$, the equations have infinite solutions unable to be calculated. Nevertheless, in a particular circumstance, since vector (signal) $x$ only has $k$ nonzero elements corresponding with vector $S$ in the transform domain, it is sparse. When the number of samples is large enough, the value of $k$ nonzero elements can be accurately reconstructed by means of the value of vector $y$ [11]. Next we will determine the number of samples, namely the value of $M$. The theory of compressive sensing reveals that, when the number of samples $M$ satisfies the following formula, high-precision reconstruction can be achieved.

$$
M \geq \delta \cdot k \cdot \log (N / k)
$$

There into, The $\delta$ is the positive constant, The $N$ is the length of the signal $x$ and $k$ is the sparseness. As is proved, when the number of the value of the acquired monitoring signals in Gaussian independent and identical distribution satisfies Formula (8), the solutions to the equations can be precisely reconstructed through finding the optimal $l_{0}$ norm solutions, that is:

$$
\mathrm{m} \text { in } \square_{S} \square_{0} \quad \text { s.t. } \quad y=\theta S
$$

There into, $\square_{s} \square_{0}$ is the $l_{l_{0}}$ norm, $\theta=A \cdot W, \theta \in R^{M \times N}$. However it is also disastrously difficult to find the solution in the approach of the minimum $l_{0}$ norm. david Donoho has proved that in the case of sparse signals, the optimal solutions of the ${ }_{l_{0}}$ norm and the $l_{1}$ norm in the target function in the constrained space border on each other. So the $l_{1}$ norm can be applied for reconstruction and optimization in engineering application, that is:

$$
\min \square_{S} \square_{1} \quad \text { s.t. } \quad y=\theta S
$$

It can thus be seen that signal CS and reconstruction aim to solve convex constrained optimization problems so as to reconstruct the original mechanical vibration signal $x$ acquired by the sensor:

$$
x=w^{-1} \cdot S
$$




\section{Design of Mechanical Vibration Signal Processing Device}

The MVSPDs primarily fulfill the functions of reconstruction of the compressed signals received, filter de-noising, vibration signal preprocessing, signal processing, extraction of the information on mechanical vibration of the monitored equipment and uploading of the data. The devices principally include the FPGA module, the DSP module, the logic control module and the power module.

\subsection{Design of the FPGA Module}

The FPGA module, in addition to receiving the mechanical vibration signals, is also in charge of caching and preprocessing of the mechanical vibration signals given out by the MT. Besides, the FPGA module also communicates with the DSP module and exports the mechanical vibration data gained through DSP module processing in the corresponding formula to the peripheral interface of the devices. The internal logic framework for the operation of the FPGA module is shown in the following Figure 3.

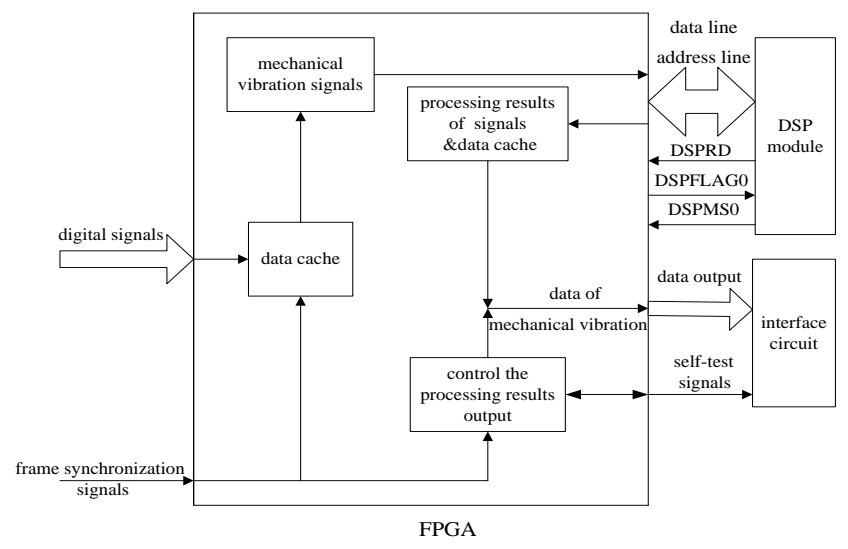

Figure 3. Schematic Diagram of the Internal Operating Logic of FPGA

The FPGA chips were used in the design by the FLASH dedicated serial configuration FLASH. This method of configuration is achievable only when the four circuits are linked, boasting the advantages of few link lines, low power dissipation and high reliability in addition to convenient internally integrated configuration. The FPGA configuration also needs the space of $16 \mathrm{Mb}$. The design adopts chip XCF32P whose storage space can meet the engineering demands [12].

The serial output of the external port should comply with the RS485 Level Standard. The design applies MAX485 to convert FPGA output into the serial RS485 level [13]. The schematic diagram of the connecting circuit is demonstrated in Figure 4.

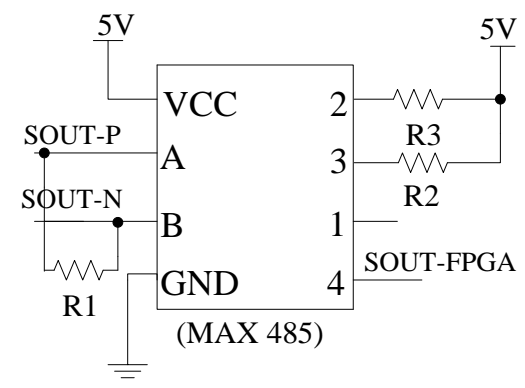

Figure 4: Conversion Circuit Schematic Diagrams 


\subsection{Design of the DSP Module}

The DSP module is the core of data processing chiefly in charge of reconstructing the algorithm of the compressed signals transferred by FPGA, extracting the vibration information to obtain the mechanical vibration information of the monitored equipment and sending back the data gained to FPGA. The framework of the hardware structure of the DSP module is shown in the following Figure 5:

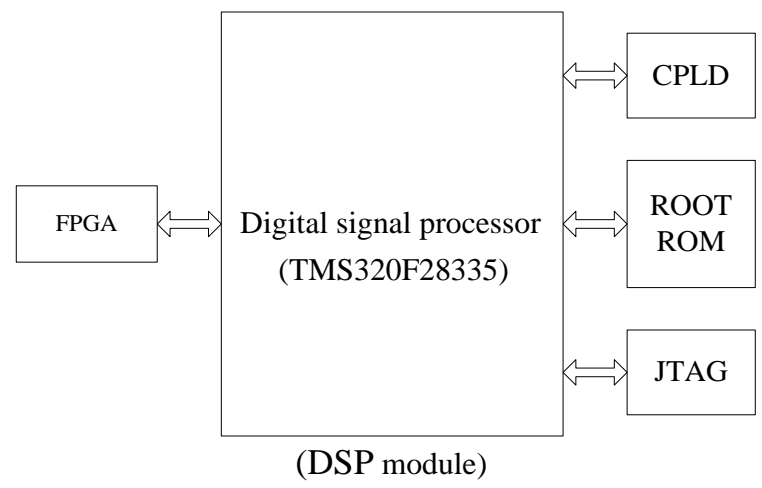

Figure 5. Hardware Structure of the DSP Module

The interface connection between the DSP module and the FPGA module includes the data line, the address line, the R\&W signals, chip select signals and interrupt signals. The connection between the DSP and the loaded FLASH includes the 16bit data line, the 20bit address line, the R\&W signals and the chip select signals.

The JTAG of the device is mainly used for internal testing of the DSP chips, monitoring of DSP and uploading the run code. The design adopts standard JTAG interfaces of the models including TD0, TD1, TMS, EMU0, EMU1 and TRST. The first four interfaces are separately utilized to test data output, data input and mode selection; EMU0 and EMU1 are analog pins; TRST is the test reset signal. The schematic diagram of the JATG interface circuit is specified in Figure 6.

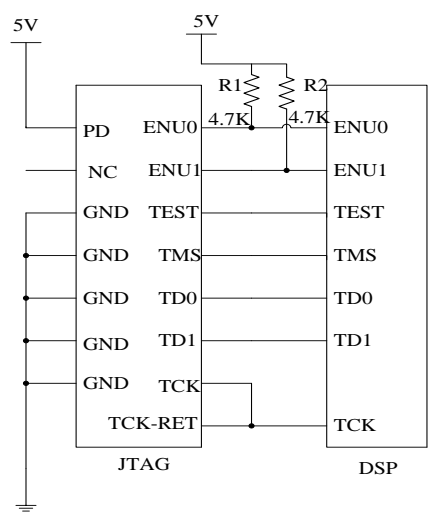

Figure 6. Schematic Diagram of JTAG Interface Circuit

\subsection{Design of the Control Module}

The system control generates the reset signal needed by the system and controls the system circuit with the structure diagram illustrated below. 


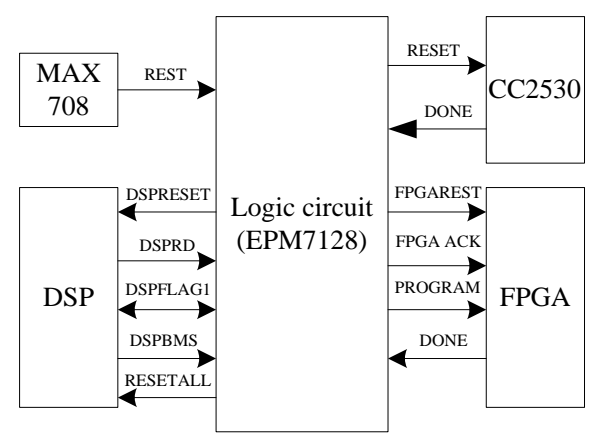

Figure 7. Logic and Control Circuit Schematic Diagram

Reset signal includes power-on reset and software reset. The former primarily comes from MAX708 and then goes through CPLD for timing control to meet the requirements of timing sequence of the reset of each part. CPLD also has the functions to control the DSP on the R\&W operation of the loaded ROM and generate the signals of system state for convenient debugging.

\subsection{Design of the Power Module}

The operating voltages of chips applied in the vibration signal processing devices include $5 \mathrm{~V}, 3.3 \mathrm{~V}$ and $1.8 \mathrm{~V}$ and the input voltage of the external power source in the design of the device is $5 \mathrm{~V}$. There into, the $3.3 \mathrm{~V}$ and $1.8 \mathrm{~V}$ are converted from the TPS767D301 chips of TI. Digital analog power source is not distinguished in the circuit and the unified ground plane is applied. The schematic diagram of the conversion circuit is manifested in Figure 8.

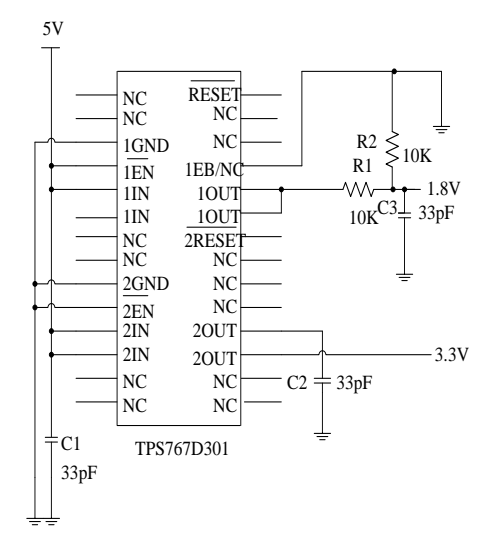

Figure 8. Schematic Diagram of Power Circuit

\section{The WSMN System Spot Test}

The WSMN is composed of the coordinator (FFD), the router and the terminal equipment (RFD) [14]. When the devices are started, the coordinator is firstly enabled to search the physical channel to make sure whether there is wireless network on the channel. If no wireless network is searched, a new process of network establishment is initiated. The network layer of the coordinator requests the MAC layer to search the energy and record the strength of the channel required in the protocol or the default physical channel. When the NLME receives the results of energy scanning, the values concerned should be sequenced from low to high. The channel of small energy values in the scope of the permitted energy level is selected, with other channels discarded. When the optimal channel of the network is selected, the new wireless network is established. After that it will choose a PAN identifier 
and a 16bit network address equal to $0 \mathrm{x} 0000$, and set the PIB property of the address of the MAC layer. When other nodes are added into the network, they will be dispatched to a 16bit network address within PAN.

When the WSMN begins to operate, the data package and the order package on all the MTs, MVSPDs and PC are swapped in the following specific process. The data acquisition software of the PC enables the acquisition task. The MVSPD synchronizes the time of all the MTs and then gives instructions. The sensors start to collect the mechanical vibration signals, compress the acquired signals and send them to the MVSPD through the wireless network. Through using restructuring algorithm and signal processing algorithms, vibration data of the monitored equipment will be obtained. The data will be saved to the SD card in the MVSPD. The MVSPD and the PC via RS485 serial interface connection, the monitoring data will be uploaded.

To verify the accuracy of monitoring data which were collected by the WSMN, using a high precision data acquisition card (HPDAC) acquires the vibration signals from the monitored equipment directly. Meanwhile using the WSMN collect vibration signals in the same equipment. After that, the two sets of vibration signals are analyzed and compared by using the same algorithm. In this way, whether the WSMN fulfils the anticipated design objectives can be reflected. In the testing, the rolling bearing of the pump in coal mine is selected as monitoring target. In the testing, the monitoring signals of 4 seconds long are intercepted. The sampling frequency is set as $1 \mathrm{kHz}$, and the number of the sampling points is 4001. By using the FFT transform, the spectrograms of two groups of the signals are obtained. The two spectrums were shown in figure 9 and figure 10.

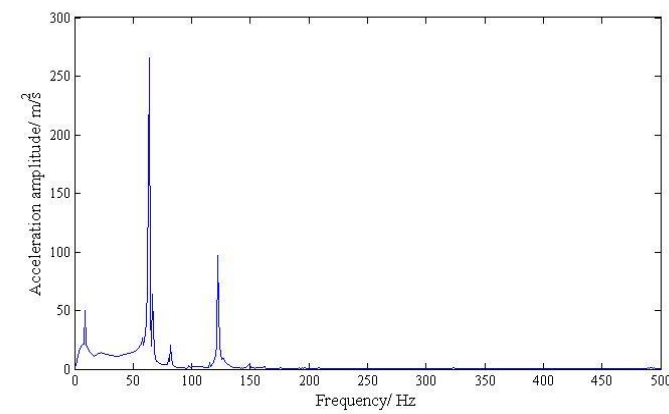

Figure 9. Vibration Signal Spectrum Collected By HPDAC

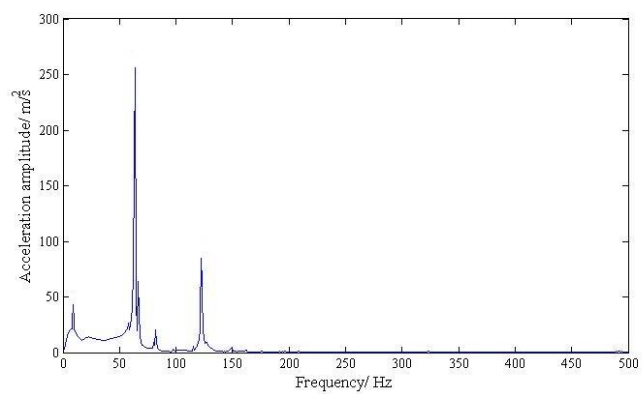

Figure 10. Vibration Signal Spectrum Collected By WSMN

Two sets of signals of several peak points of amplitude value and their error are shown by the Table 1 . 


\section{Table 1. Comparison of the Amplitude Value Error of the Frequency Peak} Points

\begin{tabular}{cccc}
\hline \multicolumn{4}{c}{ Comparison of the amplitude value } \\
\hline Peak point $/ \mathrm{Hz}$ & $\mathrm{HPDAC} / m \cdot \mathrm{s}^{-2}$ & $\mathrm{WSMN} / \mathrm{m} \cdot \mathrm{s}^{-2}$ & Error/\% \\
15.7 & 51.5 & 48.7 & 5.4 \\
67.9 & 268 & 261.5 & 2.4 \\
83.7 & 21.7 & 21.5 & 0.9 \\
128.6 & 97.1 & 88.9 & 8.4 \\
\hline
\end{tabular}

By comparing the two spectrograms, it can be seen that the spectrums of the two signals are basically similar, and the relative errors among the several major frequency points of energy focus are small. The data fully illustrate that the design goal of the WSMN is complemented well.

\section{Conclusion}

The paper described a hardware design method of wireless sensor monitoring network. Design focused on achieving the goal is to reduce the energy consumption and real-time signal processing. The WSMN's chips are likely to be low power chips, as well as special designs to reduce energy consumption. The signal processing device using the FPGA+DSP schema can achieve real-time processing of vibration signals. In addition, by using the technique of compressive sensing signal sampling and transport mode were improved, promoted transmission efficiency.

\section{Acknowledgements}

These should be brief and placed at the end of the text before the references.

First of all, I want to thank my mentor. In the aspects of hardware design and chip select, he gave me a lot of valuable advice. Secondly, I would like to thank my classmates; they assisted me in the successful completion of the test.

\section{References}

[1] Y. Dejie, C. Junsheng and Y. Yu, Journal of Vibration Engineering, vol. 3, no. 17, (2001).

[2] C. Weiwei, T. Baobing and Z. Qng-qing, Journal of vibration and Shock, vol. 1, no. 32, (2013).

[3] W. Quan, L. Fangxu and Z. Jincheng, Journal of Computer Engineering and Design, vol. 7, no. 35, (2014).

[4] L. Fangxu, Z. Jiucheng, S. Hougjun, W. Quan and W. Yu, Chinese Journal of Sensors and Actuators, vol. 10, no. 26, (2013).

[5] J. A. Gutierrez, E. H. Callaway and R. L. Barren, "Low-Rate Wireless Personal Area Networks", Enabling Wireless Sensors with IEEE 802.15.4., New York, (2003).

[6] ZigBee Aliance, ZigBee Specification. [EB/OL], http://www.ZigBee.org, (2008).

[7] Ledeczi and H. T. Volgyesi, IEEE Sensors Journal, vol. 9, no. 11, (2009).

[8] P. Junwei, Editor, "Research on wide band spectrum sensing of cognitive radios", Harbin Engineering University Publishers, Harbin, (2013).

[9] B. Lanshu, L. Yike and L. Huiyi, Journal of Geophys (in Chinese), vol. 57, no. 9, (2014).

[10] D. Donoho, "Transactions on Information Theory", Journal of IEEE, vol. 52, no. 4, (2006).

[11] Z. Bo, L. Yulin, C. Bowen and Z. Tianxin, Journal of Chongqing University of Posts and Telecommunications(Natural Science Edition), vol. 26, no. 2, (2014).

[12] H. Xiao, H. Mo and Z. Zhimin, Journal of Microcomputer Information, vol. 11, (2009).

[13] Texas Instruments Incorporated RS-485 for E-Meter Application [EB /OL]. http//focus ti com/lit/an /sl1a112a /sl1a112a pdf, (2008).

[14] P. Yan, Journal of Modern Electronics Technique, vol.34, no. 5, (2011). 


\section{Author}

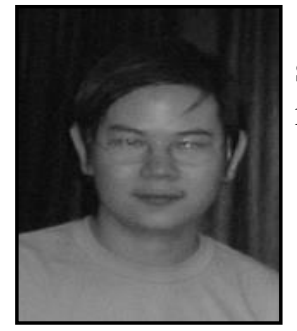

Ma Naji (1981) Male, PhDstudent, research interests: wireless sensor network, reducing network power consumption, monitoring signal processing. 
International Journal of Future Generation Communication and Networking Vol. 8, No. 5 (2015) 